\title{
Carnets
}

Revue électronique d'études françaises de l'APEF

Deuxième série - $12 \mid 2018$

Théorie Mimétique et Études Littéraires

\section{Le mythe cosmogonique de Pangu}

La fonction de la violence dans la fondation du monde

João Marcelo Mesquita Martins

\section{(2) OpenEdition}

Journals

Édition électronique

URL : http://journals.openedition.org/carnets/2486

DOI : $10.4000 /$ carnets.2486

ISSN : 1646-7698

Éditeur

APEF

Référence électronique

João Marcelo Mesquita Martins, « Le mythe cosmogonique de Pangu », Carnets [En ligne], Deuxième série - 12 | 2018, mis en ligne le 19 janvier 2018, consulté le 30 avril 2019. URL : http://

journals.openedition.org/carnets/2486; DOI : 10.4000/carnets.2486

Ce document a été généré automatiquement le 30 avril 2019

\section{(c) (i) \&}

Carnets est mis à disposition selon les termes de la licence Creative Commons - Atribution - Pas d'utilisation commerciale 4.0 International. 


\title{
Le mythe cosmogonique de Pangu
}

\author{
La fonction de la violence dans la fondation du monde
}

João Marcelo Mesquita Martins

\section{Notes brèves sur l'origine du monde}

1 Transmis d'abord sous forme orale, le mythe d'origine procure un ensemble d'idées sur la mentalité de la communauté qui l'a créé. Son développement ainsi que les changements que la société subit ont aussi une influence sur les contenus transmis par le mythe. Par conséquent, la présence dans les mythes d'origine de personnages déjà définis est tout à fait normale. Certains de ces mythes donnent une justification de ce fait. Mais il y en a d'autres qui ne le font pas. L'émergence du monde se met fréquemment en place à partir d'un cosmos plus ou moins constitué.

En racontant de quelle façon l'univers s'est organisé à partir de circonstances chaotiques, le mythe d'origine dévoile la fascination qui s'empare de plus en plus de l'être humain pour le traçage de la création et de la formation du cosmos qu'il habite. «En somme, les mythes décrivent les diverses, et parfois dramatiques, irruptions du sacré (ou du surnaturel) dans le Monde. C'est cette irruption du sacré qui fonde réellement le monde et qui le fait tel qu'il est aujourd'hui " (Eliade, 1963: 15). La recherche de réponses aux questions de la création et de la formation du cosmos favorise la formulation d'hypothèses sur l'instant où un certain élément ou une certaine substance a donné lieu à tous les autres éléments qui composent l'environnement.

$\mathrm{Au}$ commencement de la lointaine antiquité, qui en a transmis le conte?

Alors, le ciel et la terre étaient unis. Comment ont-ils été explorés?

Alors, il y avait le chaos. Qui l'a ordonné ?

Quelles choses flottaient dans l'air et comment pouvait-on les distinguer?

Il y avait le jour et la nuit est apparue. Avec quel propos?

Yin, Yang et le Ciel, quelle est la forme originale et comment s'est-elle transformée

?

Le firmament a neuf couches, administrées par qui ?

Une œuvre aussi grandiose, qui l'a créée à l'origine ? (Apud Yuan, 2006 : 18) (TdA) 
3 À titre d'exemple, le poète et ministre chinois Qu Yuan (habituallement, 340 - 278 a.C.), présente, dans son œuvre Questions au Ciel (天问, Tiānwèn), un ensemble de cent soixante douze questions en vers sur des mythes et d'anciennes croyances religieuses, présentes dans l'anthologie Chansons du Sud (楚辞, Chǔci) $)^{1}$, quelques renseignements sur la séparation du Ciel et de la Terre et l'organisation de l'Univers, tout en mettant en relief le composant primordial de ces phénomènes.

4 Selon Lamas (1991), le système mythologique chinois ne doit pas être analysé sur une seule ligne de pensée, qu'elle soit historique, sociologique ou anthropologique, car cela signifie ne pas prendre en compte que la Chine est constituée d'un mélange de peuples ${ }^{2}$, influencés surtout par le Taoïsme, le Confucionisme et le Bouddhisme, capable de superposer des éléments d'origine diversifiée et de conjuguer non seulement des figures des pensées philosophico-religieuses déjà signalées, mais aussi de vieilles divinités indigènes et des personnages que l'héroïsme a divinisés.

5 En ce qui concerne le mythe de fondation, sa conceptualisation a toujours été présente dans le tréfonds de la pensée des ethnies mentionnées. Souvent leurs récits et leurs intrigues s'entrelacent et se complexifient. Il faut souligner pour ce point spécifique la différence évidente entre les spéculations d'origine chinoise et celles d'origine judéochrétienne sur la création et la formation du monde. Au contraire de celle-ci, qui ne présente qu'un épisode fondateur, dans la tradition chinoise il n'y a pas un mythe qui raconte l'origine vraie du monde et de l'homme mais une multiplicité de mythes, parfois paradoxaux. Il faut donc parler d'un pluralisme de conceptions cosmogoniques chinoises (cf. Martins, 2015).

Dans la mythologie chinoise, l'acte de création était conçu non pas comme tirer quelque chose à partir de rien, mais de transformer l'amorphe en un ordre. L'univers a surgi quand des éléments, jusque-là indifférentiés, ont été séparés. L'ancien people chinois imaginait le chaos primordial comme un nuage de vapeur humide suspendue dans l'obscurité (Allan \& Phillips, 2012 : 30) (TdA).

6 Birrell (1993) suit Allan et Phillips quand ils suggèrent que, quoiqu'éloignés par d'innombrables barrières géographiques, beaucoup de sociétés et de cultures sont parties d'une même souche commune pour la conception de ce type de mythes. En outre, la chercheuse croit que la cosmogonie chinoise, différemment des ancestrales cosmogonies grecques, égyptiennes et mésopotamiennes, qui placent l'origine dans l'eau, pose la vapeur comme pilier primitif (气, qi), comprenant l'énergie cosmique responsable de l'ordonnancement de la matière, temps et espace. D'ailleurs, comme l'auteure le dit, « la même vapeur subit une modification au moment de la création de façon à ce que cet élément nébuleux se différentie en des éléments binaires comme masculin et féminin, Yin et Yang, matière dure et molle, entre autres " (Birrell, 1993: 23) (TdA). Cette vapeur, principe central de la médicine traditionnelle chinoise et des arts martiaux d'origine chinoise, trouve des principes équivalents dans des concepts (occidentaux) comme le pneuma, considéré dans la philosophie stoïque comme le souffle de vie qui organise l'individu et le cosmos. 


\section{La séparation du Ciel et de la Terre : l'histoire de Pangu (盘古开天辟地, Pángǔ kāitiān pidi)}

7 Dans ce contexte, bien que l'on puisse évoquer plusieurs récits, celui qui est raconté le plus fréquemment est le mythe de Pangu (盘古, Pángǔ), dont le recueil et identification des fragments datent, selon Bodde (1961), du IIIe siècle. Visiblement influencé par le développement d'un réseau d'échanges commerciaux et culturels établis sur la Route de la Soie, l'importance du mythe de Pangu est justifiée pour notre étude quand on sait qu'il a été très probablement véhiculé par des peuples exogènes à la culture chinoise.

Deux raisons expliquent pourquoi on peut conclure que ce mythe n'est pas issu de la tradition native chinoise. D'abord, le mythe n'est pas référé dans les premiers textes de la Chine classique, il n'émerge que dans des œuvres posthumes du IIIe siècle. Ensuite, ce mythe partage tant de caractéristiques avec le mythologème du corps humain cosmologique qu'il semble probable qu'il ait été emprunté (...) aux sources de l'Asie Centrale qui pénétraient alors en Chine (Birrell, 1993 : 31) (TdA).

8 L'importance de la dualité transmise par le mythe doit être également soulignée, étant donné que, lors de la séparation du Ciel et de la Terre, le sacrifice de Pangu renvoie aux concepts immémoriaux de Yin et Yang (阴阳, yinnyáng). La structuration du cosmos est réussie moyennant la conjugaison des deux réalités antithétiques, des forces opposées qui ne subsistent pas sans l'autre.

9 Le récit que l'on transcrit ci-dessous n'est qu'une des versions qui existent sur Pangu, car les détails et les particularités de chaque mythe sont fréquemment modifiés dans sa circulation par des ethnies différentes (ce qui investit son noyau d'une spécificité concrète). Celui de Pangu ne fait pas exception.

On dit que, quand le Ciel et la Terre n'étaient pas encore séparés, il n'y avait dans l'univers que chaos et obscurité comme si c'était un grand œuf. Dans cet œuf dormait profondément, grandissait l'ancestral Pangu, qui resta ainsi pendant 18000 ans. Un jour, il se réveilla soudainement, ouvrit les yeux et s'exclama: "on ne voit rien! Tout est noir, gluant et étouffant!"

Énervé et embêté, il prit une hache issue de rien et, en le brandissant avec force contre le noir chaos, Pangu lui porta un coup fracassant. Le grand œuf se brisa. Il en sortit d'une part un ensemble de choses légères et limpides qui, en montante lentement, devint le Ciel, et, d'autre part, un ensemble de choses Lourdes et troubles qui, en descendant profondément, devint la Terre. Au commencement, le Ciel et la Terre étaient unis, mais lorsque Pangu brandit la hache vigoureusement, ils se sont séparés et différentiés. Après cette séparation, Pangu craignait que le Ciel et la Terre ne s'unissent de nouveau. Alors, en se mettant entre les deux, il utilisa sa tête comme support du Ciel et foula la Terre avec les pieds. Au fur et à mesure que Ciel et Terre changeaient, lui-même se transfigurait. Chaque jour le Ciel montait un zhang chinois, la Terre grossissait un autre zhang et le corps même de Pangu augmentait pareillement ${ }^{3}$. Encore 18000 années s'écoulèrent : le Ciel de plus en plus élevé, la Terre de plus en plus grosse et le corps de Pangu de plus en plus long.

Quel était la longueur du corps de cet être ? On estime qu'il atteignit une longueur de 90000 li chinois ${ }^{4}$. Cet être majestueux ressemblait à une colonne entre Ciel et Terre, qui empêchait qu'ils reviennent de nouveau au chaos.

Pendant longtemps, Pangu exécutait tout seul ce travail de séparation du Ciel et de la Terre. À la fin, la structure établie entre eux devint assez solide pour que l'ancêtre cesse de s'inquiéter d'une possible réunion. À vrai dire, il avait besoin de se reposer. Tout comme les humains il tomba mort.

Au moment de mourir, son corps se transfigura : l'air qui sortait de sa bouche se 
transforma en des nuages et du vent ; la voix créa la foudre ; l'œil gauche devint le soleil et l'œil droit la lune; les membres et le corps devinrent les quatre pôles de la terre et les cinq montagnes ${ }^{5}$; le sang forma les fleuves et les veines les routes; les muscles devinrent les champs pour l'agriculture ; les cheveux et la barbe montèrent au Ciel et devinrent les étoiles; la peau et l'autre pilosité donna forme aux fleurs, herbes et arbres; les dents, les os, la moelle osseuse, entre autres, se transformèrent en des métaux luisants, des pierres solides, des perles rondes et brillantes et en jade délicat; et, finalement, la sueur, qui au début n'avait aucun usage, se modifia en rosée et pluie. En bref: Pangu, qui mourut et se transforma, usa tout le corps pour que ce monde nouveau devienne plus riche et plus beau (Yuan, $2006: 26 / 27 / 28$ ) (TdA).

Bodde (1961) pense que le récit autour de Pangu est une compilation d'information fragmentée et datée. Selon l'auteur, le mythe de Pangu n'est pas originaire de l'ethnie Han, au contraire, il a un lien plus fort avec quelques ethnies du Sud de la Chine, dans lesquelles on raconte l'histoire d'un chien appelé Pan'hu (槃瓠, Pánhù). Après avoir tué, décapité et apporté la tête d'un chef rebelle sous l'ordre de l'Empereur $\mathrm{Ku}$, son propriétaire, Pan'hu obtint le droit d'épouser sa fille. Dans le Sud de la Chine, le couple et sa descendance sont tenus pour des ancêtres de certaines ethnies, comme la Bai ou la Zhuang. Outre le fait que les deux mythes sont censés être apparus dans le Sud du pays, la ressemblance phonétique entre Pangu et Pan'hu fonde un rapport possible entre eux.

11 Pourtant, l'aspect le plus intéressant de ce mythe est sans doute la similarité qu'il présente avec d'autres mythes de la même catégorie. L'origine cosmique assumée par le corps humain est évidente dans l'extrait cité : le macro-organisme, le monde, est créé par la division du micro-organisme, le corps de Pangu, dont chaque partie correspond à une section, pour ainsi dire, du cosmos engendré.

Bien que le mythe de Pangu n'arrive en Chine qu'au IIIe siècle, Eberhart (...) l'a mis en rapport conceptuel avec une idée chinoise bien plus ancienne : celle de l'œuf ou sac primordial dont la rupture permet à ses éléments indifférenciés de prendre la forme d'un univers organisé. Dans sa version sophistiquée, cette conception peut très bien constituer la base de la théorie astronomique, qui circulait au temps de la dynastie Han, selon laquelle le Ciel et la Terre ont la forme d'un œuf. La Terre est enclose dans la sphère du Ciel tout comme le jaune d'œuf est enclos dans sa coquille (Bodde, 1961 : 384) (TdA).

12 A ce point spécifique, il est possible d'établir une liaison avec le mythe d'origine hindou. L'utérus cosmogonique, en tant qu'élément d'où est issu Pangu et donc le monde, est un motif que l'on trouve aussi dans la trinité suprême de l'Hindouisme, constituée de Brahma (le Dieu Créateur), Vishnou (le Dieu Conservateur) et Shiva (le Dieu Destructeur). Ling conjecture que, parmi les hymnes du dixième hymne du Rig-veda, mérite une attention particulière le X.90 « Le Sacrifice du Premier Homme ». Il affirme :

(...) l'origine de l'univers est présentée comme un processus sacrificiel. Ce que l'on peut identifier ici, c'est l'élévation de l'acte sacrificiel même à une position d'extrême importante dans le schéma religieux, de façon à devenir encore plus important que les dieux à qui le sacrifice était offert. D'où découle la notion de sacrifice, le processus qui a fait naître l'univers, comme un facteur vraiment efficace. (...) L'accent mis sur l'importance de l'activité sacrificielle a relégué les dieux à une position inférieure dans le schéma religieux des choses, en même temps que le sacrifice a été investi d'une certaine dimension magique. Il est devenu l'élément vital du mécanisme cosmique; la préservation de l'ordre cosmique était vue maintenant comme étant dépendante de l'observation du protocole du rituel sacrificiel (Ling, $1994: 67)$. 
13 Le sacrifice du premier homme, à son avis, permet une justification de l'émergence du système de castes. Selon Zaehner, à la question « Quand le premier homme a été divisé, en combien de parties a-t-il été divisé ?", il répond «Le brahmane était sa bouche, les bras sont devenus le prince (ksatriya), les cuisses le peuple (vaiśya) et des pieds est sorti le serf (sudra) » (Apud Ling, 1994 : 68). Lü (1941) reconnait également que de Brahma sont issues les castes qui composent actuellement la société indienne : de la bouche est issue la caste des prêtres dont l'objectif serait la célébration liturgique; des bras est sortie la caste des guerriers; des cuisses, la caste des marchands et des agriculteurs; et, finalement, des pieds a émergé la quatrième caste : celle des artistes et ouvriers de toute espèce (serfs). En plus, la symbologie associée aux trois divinités est importante pour la présente étude, étant donné que le renouvellement cosmologique hindou est cyclique. Le cosmos, détruit par Shiva, revient au chaos initial, à l'océan primordial où somnole Vishnou. Quand la reprise du cosmos est imminente, Brahma, sortant d'une fleur de lotus ${ }^{6}$ qui jaillit du nombril de Vishnu, rend tangible cet événement.

On peut en conclure que les ressemblances entre les mythes décrits doivent découler de l'échange culturel intense qui reliait autrefois les peuples de l'Asie Centrale. La création de castes et la récréation du cosmos sont assez proches de la fondation du monde par Pangu, puisque toutes trois éliminent le chaos sous le mode d'obscurité ou de désorganisation sociale et permettent l'entrée dans un système ordonné, dans une nouvelle réalité.

Finalement, dans une autre perspective, notamment celle du sacrifice fondateur, une comparaison entre Pangu et Jésus Christ devient viable. Dans le mythe chinois, Pangu sacrifie sa vie même pour éviter la réunion du Ciel et de la Terre, offrant son corps physique pour transformer la Terre dans un être vivant. La connexion entre les entités et leurs pouvoirs cosmiques respectifs permet la métamorphose de Pangu et la formation du monde qui en résulte. Jésus Christ, dans la tradition judéo-chrétienne ${ }^{7}$, sert comme expiation pour le salut de l'humanité lorsqu'il est sacrifié sur la croix. Accusé et jugé par ses actes, Jésus Christ ne se défend pas, puisqu'il est l'agent, envoyé par Dieu, du salut, moyennant la rédemption des péchés de l'humanité. Les deux figures se sacrifient consciemment et volontairement pour empêcher la récidive chaotique (cf. Martins, 2015).

\section{Le rôle de la violence dans la fondation du monde}

Girard soutient une vision particulière sur la façon dont les mythes doivent être analysés à partir d'une théorie du désir. À l'opposé de la conception romantique, Girard développe une conception mimétique du désir qui le définit comme fondamentalement aliéné puisque déterminé par le désir d'autrui : je viens ainsi à désirer ce que l'autre désire. Il observe les profondes rivalités qui découlent de la structure triangulaire du désir impliquant sujet, modèle-rival et objet et qui aboutissent à la violence collective.

Dans le déroulement du processus, cette rivalité se radicalise si bien que la violence s'incorpore au noyau du désir même. Il semble donc que la violence devient l'objet du désir, le sujet $A$ voulant maîtriser l'objet moyennant la violence contre le sujet $B$. L'ensemble de ce processus est la crise mimétique : les deux sujets ne désirent pas l'objet mais le désir de l'autre. À ce moment-là l'objet a disparu derrière le désir réciproque. Ou plutôt: objet, sujet et désir ne font qu'un; ils sont indifférenciés. Cette indifférenciation est à l'origine de la violence. (...) La structure du désir est la structure de la violence (Schultz, $2004: 11$ ). 

dans les sociétés archaïques et anciennes. Comment ces sociétés dépourvues de dispositifs institutionnels judiciaires ont-elles fait face aux revanches, aux conflits sociaux et autres formes de la violence mimétique qui les détruisaient? sociétés humaines et dont il décrit le fonctionnement selon ce qu'il appelle le mécanisme du bouc émissaire ou mécanisme victimaire.

Ce sont les rivalités mimétiques qui le [le mécanisme du bouc émissaire] suscitent en se polarisant spontanément, à leur, paroxysme, contre une victime unique. L'exécration et la destruction unanimes d'un pseudo-ennemi réconcilient la communauté, aux prix relativement modique d'une seule victime. Le phénomène est d'autant plus précieux que les communautés parviennent à le reproduire sur des victimes de rechange : c'est le sacrifice rituel (Girard, $2003: 48$ ).

Le sacrifice, qui institutionnalise le mécanisme victimaire, est la solution mise en place par les sociétés archaïques pour rationaliser et domestiquer la violence intestine. Il permet à une communauté de canaliser la violence réciproque et acéphale vers un seul objet et lui donner une direction. En effet, «la menace collective est condensée en une seule victime ; du menaçant "l'un contre l'autre" on passe à l'apaisant "tous contre un" " (Schultz, 2004 : 11). Institution de fond d'où dérivent toutes les autres, le sacrifice opère un double déplacement: la violence est condensée dans la haine unanime contre la victime, ce qui permet au groupe de la dissimuler et de se réconcilier. Auparavant coupable de tous les maux, la victime acquiert une dimension transcendante et est vue comme source de sacré et de salut.

La violence du sacrifice produit le sacré et sacralise la violence. Chassée de la société pour ses effets maléfiques, la violence fondatrice est en même temps vénérée pour sa vertu bénéfique. La victime se trouve dans une sphère ambiguë entre le bien et le mal. Elle naît de l'indifférenciation et produit la différenciation; elle fonde la culture. Elle est maléfique dans la mesure où elle condense la méchanceté sociale en tant que bouc émissaire, mais elle est rédemptrice car elle libère les persécuteurs de leurs récriminations réciproques et apporte des bienfaits sociaux (Schultz, 2004 : 13) (TdA).

Donc, pour Girard, désir, rivalité et crise mimétique et leur résolution au travers du bouc émissaire font partie du mécanisme victimaire comme un tout. Quand la violence s'accumule dans un certain groupe social, ce mécanisme évite la destruction dudit groupe. Aussi fonde-t-il la religion, outre la culture.

21 L'étude des mythes est une partie très importante de l'œuvre de Girard. Selon Millán Alba (2008), il identifie dans le mythe : la structure du désir mimétique qui conduit à vouloir ce que les autres veulent, aboutissant à la violence généralisée ; la structure de la violence et $\mathrm{du}$ meurtre fondateur qui coïncide avec celle du parricide primitif chez Freud; la résolution de la violence à travers le sacrifice, c'est-à-dire l'immolation d'un individu qui assume le rôle de bouc émissaire; et la structure de la sacralité causée par la violence sacrificielle et sa perpétuation (liturgique) dans le rite.

Après avoir oscillé entre les trois protagonistes, l'accusation décisive finit par se fixer sur l'un d'entre eux. Elle aurait pu aussi bien se fixer sur un autre. Elle aurait pu ne pas se fixer du tout. (...) L'accusation qui va désormais passer pour "vraie ", ne se distingue en rien de celles qui vont désormais passer pour fausses, à ceci près qu'aucune voix ne s'élève plus pour contredire qui que ce soit. Une version particulière des événements réussit à s'imposer ; elle perd son caractère polémique pour devenir la vérité du mythe, le mythe lui-même. La fixation mythique doit se 
définir comme un phénomène d'unanimité. Là où deux, trois, mille accusations symétriques et inverses se croisaient, une seule l'emporte et autour d'elle tout se tait. L'antagonisme de chacun contre chacun fait place à l'union de tous contre un seul (Girard, $1990: 120$ ).

À l'origine des mythes, qu'ils soient plus primitifs ou plus complexes, il y a d'abord un état de confusion cosmique et sociale. L'indifférenciation, considérée dangereuse, fait que les hommes et les animaux ne se distinguent pas les uns des autres. Dans les mots de Millán Alba (2008: 79), "l'indifférenciation règne partout », presque transmise à la nature. L'idée de chaos original est présente dans quasiment toutes les cosmogonies, où des êtres indifférenciés luttent les uns contre les autres pour se différencier.

Le mythe introduit une restructuration de la culture quand elle est dominée par le désordre original. Cette restructuration ne devient possible que quand le sommet du désordre indifférencié est atteint. Girard attribue cette fonction ordonnatrice au bouc émissaire, car c'est lui qui unifie de nouveau la société et lui rend l'essence perdue. La violence aboutit toujours à un retour à l'origine sacrée. Autrement dit, c'est la violence elle-même, représentée dans le sacrifice de la victime collective, qui paradoxalement se convertit en sacré, car le sacrifice du bouc émissaire est la matrice du rite qui l'actualise et perpétue, déclenchant une sorte de catharsis communautaire. Ceci est le dernier pas dans la structure mythique analysée par Girard: la divinisation du bouc émissaire et sa réactualisation par le biais du rite. Von Baltasar croit que

Le schéma primitif de l'immolation du bouc émissaire se trouve à la base de tous les mythes, de façon plus ou moins voilée, mais aussi, ce qui est le plus important, à la base de tous les rites, car effectivement le rite est la régulation originale d'une "crise sacrificielle" qui se répète périodiquement quand un groupe, après une phase à peu près calme, lutte pour se déprendre et émerger dans une nouvelle période de violence mimétique. Alors le rite offre la solution suivante : l'élection unanime d'une victime pour l'offrir à la divine et féroce violence (au début la victime humaine, puis une victime animale le plus proche possible de l'homme). Tous les rites, y compris tous les interdits (inceste) et prescriptions rituelles, même le canibalisme, renvoyent à la répétition cathartique du drame du bouc émissaire. ( Apud Millán Alba, 2008 : 83/84) (TdA)

\section{Brève analyse du cas chinois}

Beaucoup de cultures ont développé des récits mythiques sur des figures divines dont la mort est essentiellement constitutive de la réalité. Par exemple, Eliade croit que ce type de mythe est très commun dans les sociétés qui produisent des céréales, vu que «la période embryonnaire du futur souverain correspondait au processus de maturation de l'Univers et, très probablement, était originellement en relation avec la maturation des récoltes " (Eliade, 1963: 54). Le mythe chinois, le mythe védique et le mythe nordique ${ }^{8}$, par exemple, présentent le récit d'un géant cosmique qui organise le chaos, l'annulant et le transformant en cosmos. Le dessein de la mort de Jésus Christ, en tant que sauveur qui prend sur soi les fautes d'autrui, est un autre exemple qui intéresse ce type de récits.

(...) Il s'agit de rituels collectifs d'une périodicité irrégulière, comportant la construction d'une maison cultuelle et la récitation solennelle des mythes d'origine de structure cosmogonique. Le bénéficiaire en est l'ensemble de la communauté, les vivants comme les morts. A l'occasion de la réactualisation des mythes, la communauté tout entière est renouvelée ; elle retrouve ses "sources", elle revit ses "origines". L'idée d'un renouvellement universel opéré par la réactualisation 
cultuelle d'un mythe cosmogonique est attestée chez beaucoup de sociétés traditionnelles (Eliade, $1963: 50$ ).

Pour autant que le récit mythique chinois ne peut pas être un objet direct et net de la théorie de René Girard, la présente analyse vise à bâtir un pont entre le récit et la théorie. Dans le mythe de Pangu, le triangle mimétique n'est pas un élément qui possède une présence manifeste dans la mesure où Pangu, lorsqu'il est né, perçoit que le monde autour de lui est désorganisé, dans un état de complet chaos. Le chaos peut être considéré le résultat d'une triangulation antérieure. Pour cette analyse, peu importe qui a imité qui ou qui a désiré quoi. Ce qui est important c'est que, à un moment donné, un certain sujet a désiré le même objet désiré par un certain modèle. La rivalité ainsi déclenchée est une rivalité généralisée, vu que, selon Girard, le sujet qui copie le modèle est copié par un autre sujet. Cela étant, cette chaîne qui relie des sujets et des médiateurs, impliqués en des conflits directs et oublieux de l'objet d'abord désiré, conduit la société à un état de violence universelle.

Selon la théorie de Girard, le sacrifice du bouc émissaire est nécessaire à ce que la violence totale soit provisoirement abolie. Néanmoins, on considère que, avant que la figure de Pangu n'apparaisse, il n'y a pas de victime qui soit choisie par la communauté comme cible spécifique de cette violence et réussisse à parer à l'état social chaotique. Par conséquent, avant la naissance du géant, la société succombe à la violence de tous contre tous et ainsi se déplace d'un cosmos organisé à un chaos indéfini. Dans cette perspective, le noir que Pangu nouveau-né perçoit est une image métaphorique de la désorganisation sociale qui, à une époque donnée, aura affecté la société chinoise. Une autre interprétation possible dira que le fait qu'une société a été capable de choisir une victime sacrificielle pour la violence généralisée, ne signifie pas forcément qu'elle soit capable de procéder au renouvellement continu du sacrifice dans le rite. Par conséquent, des rivalités mimétiques qui pourraient être supprimées, surgissent à nouveau dans la société et poussent la communauté vers l'état qu'elle fuit.

27 Notre analyse se focalise maintenant dans l'état chaotique de la société, le noir immense qui relève d'une violence antérieure à la naissance de Pangu. Comme on l'a déjà vu, c'est cette figure mythique qui donne une nouvelle origine à la réorganisation de la société. Autrement dit, c'est à travers la mort de Pangu que la société peut récupérer son état cosmique de paix. La question à poser maintenant est de savoir pourquoi Pangu a été choisi comme victime sacrificielle. Soulignons que, quand on parle de bouc émissaire, celui-ci doit forcément avoir un ensemble de traits qui permettent le remplacement sacrificiel, comme le degré de proximité, ou plutôt, la distance que la victime présente par rapport à la société. Dans cette ligne de pensée, Girard considère que plus la victime est éloignée de la société, plus parfaite elle est pour la réalisation de l'acte sacrificiel. Aussi étrangers, prisonniers de guerre et enfants sont-ils des victimes sacrificielles prioritaires. Or, Pangu est né. C'est précisément cette naissance qui, en le réveillant à la réalité chaotique, le pose en premier dans l'éventail des choix sacrificiels, car son rapport à la société antérieure à la généralisation de la violence est en effet très mince.

Pourtant, le sacrifice de Pangu n'est pas un sacrifice mortel. Entre le moment où il est né et celui où il est mort, Pangu accomplit sa tâche pendant 18000 ans, ce qui indique la distance temporelle entre l'instant du premier sacrifice et sa finalisation. C'est-à-dire que l'état chaotique de la société est tellement grave que la mort immédiate du bouc émissaire ne réussit pas à restituer à la société la paix d'autrefois. Séparer le ciel de la terre compose ainsi une image de rite qui, constamment accompli par Pangu, lui rend le 
statut de victime sacrificielle. Ce qui est clé pour la société est alors le constant renouvellement du rite et non son exécution intégrale. Le sacrifice de Pangu devient sacré, parce qu'il réorganise le chaos, en l'annulant continument ${ }^{9}$. La mort de Pangu symbolise le moment où, la rivalité de tous contre tous étant résolue, le cosmos peut prendre sa pleine forme. Le démembrement du corps représente ainsi son intégration absolue dans la société qu'il sauve. Avant victime coupable de l'état de la société, Pangu joue un nouveau rôle, celui du sauveur, qui mérite la dévotion de la société sauvée qui se manifeste à travers le rite.

La mort de Pangu présuppose aussi que le rite qui rappelle et célèbre ce sacrifice sauveur sera un jour oublié et empêché de réaliser son propos. Ceci équivaut à dire que, à un certain point dans l'avenir, la même crise aura lieu. On devine donc une série de crises cycliques. Il est curieux de penser que, tout au long de l'histoire chinoise, plusieurs dynasties ont été établies successivement, dans un processus où les empereurs vaincus ont été pris pour des coupables de tous les maux. Il y a donc une recherche constante du renouvellement des méthodes utilisées pour mettre la fin aux rivalités mimétiques et aux crises qui en résultent. En bref, le mythe est nettement métaphorique et changeable. Nous croyons qu'il y a eu une communauté, avant Pangu, qui, pour des raisons indéterminées, a connu une période de grandes difficultés. On ne sait pas si Pangu a été un roi ou un fonctionnaire d'État ${ }^{10}$, mais il a sûrement été un personnage qui a puissamment contribué à la solution des problèmes sociaux de cette époque-là. Cela étant, il a gagné une place centrale dans la communauté : celle de l'éternel sauveur qui a réorganisé le monde.

\section{Considérations finales}

Symbole vivant, le mythe ne peut avoir une définition fermée dans la mesure où il est toujours ouvert à de nouvelles lectures. Le mythe change avec la réalité. Qu'il y ait corrélation ou disparité entre les mythes qui en découlent, les formulations expriment l'exclusivité culturelle des peuples. Cela dit, le mythe d'origine n'est pas seulement un, unique et bien défini. Au contraire, il y en a plusieurs qui partent à la recherche d'une réponse. Le mythe de Pangu, peut-être le plus intéressant, grâce à la tradition herméneutique qui s'est déployée autour de lui, rend possible une vision plus nette de la façon dont le peuple chinois perçoit le monde. Pangu est né, il sépare le ciel et la terre et, à la fin, il est mort, son corps donnant lieu aux fleuves, montagnes, pluies, plantes, êtres vivants, etc. La réflexion de Girard sur l'interaction reliant la condition humaine et la fonction centrale du désir détermine la thèse selon laquelle au fondement de nos rapports il y a toujours la nature mimétique du désir. De ce désir naîtront des rivalités et des conflits. Girard a élaboré cette théorie pour des sociétés dont le système formel de contention de la violence était encore inexistant. En introduisant le mécanisme du bouc émissaire, l'auteur perçoit le mythe comme quelque chose qui fonctionne selon la structure de la crise mimétique, meurtre fondateur et restitution de la différentiation (c'est-à-dire la société passe du chaos à l'ordre). Notre analyse a appliqué cette théorie au mythe d'origine de Pangu, dans lequel nous avons identifié le meurtre fondateur et la mise en place de la différentiation. 


\section{BIBLIOGRAPHIE}

ALlan, Tony \& PHILIPS, Charles (2012). Ancient China's Myths and Beliefs. New York: Rosen Publishing.

BIRRELL, Anne (1993). Chinese Mythology: An Introduction. Baltimore: The Johns Hopkins University Press.

BODDE, Derk (1961). « Myths of Ancient China », in Samuel Noah Kramer, Mythologies of the Ancient World. New York: Anchor Books, pp. 369-408.

ELIADE, Mircea (1963). Aspects du mythe. Paris : Gallimard.

GIRARD, René (1982). Le bouc émissaire. Paris : Grasset et Fasquelle.

GIRARD, René $(1990$; 1972). La violence et le sacré. Paris : Hachette.

GIRARD, René (2003). Le sacrifice. Paris : BNF.

GIRARD, René $(2010,1961)$. Mensonge Romantique et Vérité Romanesque. Paris : Fayard/Pluriel.

LAMAS, Maria (1991). Mitologia Geral : o Mundo dos Deuses e dos Heróis (Vol. 2), (3ª ed.). Lisboa:

Estampa.

LINCOLN, Bruce (1986). Myth, Cosmos, and Society: Indo-European Themes of Creation and Destruction. Cambridge, Mass. : Harvard University Press.

LING, Trevor (1994). História das Religiões. Lisboa : Presença.

LÜ, Simian, 吕思勉 (1941). 盘古考 (Pángǔ kăo), Estudos sobre Pangu in Ma Changyi, 中国神话学文 论选萃 (Zhōngguó shénhuàxué wénlùn xuăncui), Recortes Literários sobre Mitologia Chinesa (Vol. 1), (pp. 479-485), Pequim : 中国广播电视出版社 (Zhōngguó guăngbò diànshì chūbănshè) Imprensa de Rádio e Televisão Chinesas.

MARTINS, João Marcelo Mesquita (2015). Uma Visão Comparada sobre Diferentes Cosmogonias, Dissertação de Mestrado, Universidade do Minho, Braga, Portugal.

MILlAN ALBA, José Antonio de (2008). «Los Mitos según René Girard », Amaltea. Revista de mitocrítica , (0), pp. 63-86.

schultz, Adilson (2004). A Violência e o Sagrado segundo René Girard, Protestantismo em Revista Revista do Núcleo de Estudos e Pesquisa do Protestantismo (NEPP) da Escola Superior de Teologia, 2, 8-18.

YUAN, Ke, 袁珂 (2006). 中国古代神话 (Zhōngguó gǔdài shénhuà), Mitos da China Antiga. Pequim: 华夏 出版社 (Huáxià chūbănshè) Editora Huaxia.

\section{NOTES}

1. Anthologie constituée par seize chapitres, normalement attribuée à Qu Yuan. Il s'agit d'un ouvrage originaire du Royaume de Chu (楚国, Chŭguó), état qui se trouve au sud du Fleuve Jaune, célèbre par son exotisme. L'œuvre a innové au niveau de l'écriture poétique et a introduit une nouvelle division métrique ainsi que le caractère xi (兮, xi) comme indicateur du mode. Elle a 
permis d'approfondir la connaissance de la culture de Chu qui croyait à la sorcellerie et pratiquait des sacrifices. C'est le recueil le plus ancien de mythes archaïques, ce qui l'investit d'un statut de modèle et de référence pour l'analyse comparative et contrastive du texte mythique.

2. Dans le cas chinois, il y a 56 ethnies qui, au niveau culturel et linguistique, présentent des caractéristiques différentes. L'ethnie Han correspond à environ $92 \%$ de la population totale, les autres $8 \%$ correspondant aux 55 ethnies qui restent, par exemple l'ethnie Zhuang, la Mandchoue ou la Tibétaine.

3. Le zhang chinois est une mesure de longueur encore utilisée en Chine et qui équivaut à peu près à 3,3 mètres.

4. Le li chinois est une autre mesure de distance utilisée en Chine, qui équivaut à 500 mètres.

5. En Chine ancienne, on croyait qu'il y avait cinq montagnes sacrées, qui représentaient les quatre points cardinaux autour du point central.

6. À remarquer que le lotus, plante très valorisée dans la culture chinoise, sort de la boue (chaos) et éclot en une très belle fleur. C'est la fleur du cosmos, de la création de l'Univers.

7. À remarquer que Girard distingue le mythique et l'évangélique, cette distinction s'organisant autour du point de vue : le point de vue du groupe (persécuteurs) définit le mythe, le point de vue de la victime définit les Évangiles. Dans les mythes, la foule croit que la victime est coupable de la crise, ce qui justifie son sacrifice et l'efficacité sociale de celui-ci. Par contre, dans les Évangiles, la Passion du Christ est rapportée comme une persécution, ce qui permet de mettre au clair l'innocence de la victime. Aussi Girard a-t-il critique l'idée d'une mythologie judéo-chrétienne.

8. Birrell (1993) s'inspire de l'étude de Bruce Lincoln (1986), pour affirmer que, dans le cas de la mythologie nordique, il est possible de de comparer Pangu et le géant Ymir, lequel curieusement sort de la vapeur remplissant l'abîme sans fond qui était le chaos. Tué par les dieux, il a donné lieu, tout comme Pangu, à l'univers moyennant la division des parties de son corps. De sa sueur sont sortis les humains et les autres créatures.

9. Ici il faudrait peut-être évoquer la théologie chrétienne du salut à travers la mort et la résurrection, étant donné qu'elle implique que la restauration et la préservation de l'ordre, loin d'avoir été accomplies à un certain moment, sont des processus continus.

10. Bodde (1961) attire l'attention sur le fait que les mythes subissent l'action de l'évhémérisme, théorie qui soutient que les personnages mythiques sont à l'origine des êtres humains qui ont été divinisés soit par peur soit par révérence. Dans une perspective quelque peu différente, Girard soutient qu'à l'origine du mythe il y a une victime réelle et une persécution réelle et propose de lire les mythes comme des textes de persécution historiques (cf. Girard, 1982 : 37-69). Dans le cas chinois c'est le contraire qui se passe : les personnages mythiques subissent une démystification et deviennent des personnages historiques. Bien que le mythe ne soit pas histoire, il est possible qu'il soit son ombre. En tenant compte des origines du mythe de Pangu, il est probable qu'il ait été une figure historique de la région et pas seulement un personnage mythique.

\section{RÉSUMÉS}

En constatant le rôle axial du désir dans la condition humaine, Girard soutient que les rapports humains obéissent à la nature mimétique du désir, si bien qu'il est possible de les formaliser par un triangle mimétique dont les sommets sont occupés par sujet, modèle et objet. Des interactions ainsi établies surgissent des rivalités lesquelles ont produit dans les sociétés primitives de grands 
conflits qui ont conduit au chaos. Le bouc émissaire est le mécanisme que les sociétés primitives ont mis en place pour maîtriser la violence mimétique. Girard théorise le mécanisme du bouc émissaire cherchant à identifier dans les mythes les indices de la crise mimétique et son issue dans le meurtre fondateur. Nous nous proposons d'analyser le mythe cosmogonique de Pangu par le biais de la théorie du désir mimétique et du mécanisme du bouc émissaire en essayant d'y repérer la crise mimétique, l'homicide fondateur et la reconstruction de la différentiation dans la société de l'époque.

Noting the key role that desire holds in the human condition, Girard believes that, in human relationships, there is an imitative character of desire. It is therefore possible to draw a mimetic triangle, with subject, object, model on its vertices. From the established interactions, rivalries arose in a way that, in primitive societies, created great conflicts which led to social chaos. The scapegoat was thought as a solution to control mimetic violence in these contexts. In his work, Girard formally introduces the scapegoat mechanism, seeking to identify the structure of mimetic crisis, founding homicide in mythology. This paper proposes an analysis of the chinese cosmogonic Pangu myth through the theory of mimetic desire and the scapegoat mechanism, also trying to identify the same mimetic crisis, leading to widespread violence, the presence of the founding homicide, and the reconstruction of differentiation in the society of the mentioned period.

31 janvier 2018

\section{INDEX}

Keywords : cosmogony, mimetic desire, violence, scapegoat, Pangu

Mots-clés : cosmogonie, désir mimétique, violence, bouc émissaire, Pangu

\section{AUTEUR}

\section{JOÃO MARCELO MESQUITA MARTINS}

Universidade do Minho

jmarcelommartins[at]ilch.uminho.pt 